\title{
Cutoff periods of magnetoacoustic waves in the solar atmosphere
}

\author{
J. Kraśkiewicz ${ }^{1}$, K. Murawski ${ }^{1}$, and Z. E. Musielak ${ }^{2,3}$ \\ 1 Group of Astrophysics, UMCS, ul. Radziszewskiego 10, 20-031 Lublin, Poland \\ e-mail: kraskiew@umcs.pl \\ 2 Physics Department, University of Texas at Arlington, Arlington, TX 7019, USA \\ 3 Kiepenheuer-Institut für Sonnenphysik, Schöneckstrasse 6, 79104 Freiburg, Germany \\ Received 8 April 2018 / Accepted 28 January 2019
}

\begin{abstract}
We perform numerical simulations of magnetoacoustic waves (MAWs) in the solar atmosphere, which is gravitationally stratified and structured by either vertical or horizontal uniform magnetic fields. These waves are excited by a monochromatic driver that operates in the photosphere. We show that the gradients of the atmospheric parameters lead to filtering of the waves through the solar atmosphere and to variations of the dominant wave period with height. We use these variations to determine a local cutoff period, which shows a good agreement with the previously obtained analytical and numerical results in an isothermal solar atmosphere. In our numerical simulations, the propagation of MAWs in a more realistic model of the solar atmosphere is considered, and the obtained results demonstrate that the waves with periods higher than a local cutoff wave period are strongly reflected and become evanescent with height, while the waves with shorter wave periods are propagating, and may even reach the solar corona. Some of the evanescent waves may also tunnel and reach the atmospheric heights that would not be otherwise accessible to them. An important result of our study is excitation of chromospheric oscillations with periods equal to the period that is comparable to the observed solar chromospheric oscillations. Implications of our theoretical predictions are discussed.
\end{abstract}

Key words. Sun: atmosphere - Sun: oscillations - methods: numerical

\section{Introduction}

The solar atmosphere supports a variety of wave phenomena ranging from acoustic-gravity waves in magnetic-free regions, to magnetohydrodynamic (MHD)-gravity waves in magnetic regions. In a uniform medium, the following types of MHD waves can be identified: magnetoacoustic (fast and slow) waves (MAWs), Alfvén waves, and entropy mode (e.g., Osterbrock 1961; Rossi \& Olbert 1970; Parker 1979; Thomas 1983; Roberts 2006; Priest 2014). The stratification and temperature gradients in the solar atmosphere affect the characteristic speeds of the wave, and gradients of the wave speeds may be responsible for partial or total wave reflection (e.g., Leroy 1981; Thomas 1982, 1983; Zhugzhda \& Dzhalilov 1984a,b,c; Campos 1986; Musielak 1990). As a result of strong reflection, the waves become evanescent and they do not transfer energy any longer.

Following the early work of Osterbrock (1961), Parker (1964), Kuperus (1966), and Ulmschneider \& Stein (1982) argued that the efficiency of generation of MAWs in the solar convection zone should significantly increase with increasing magnetic field strength. Studies of excitation of MAWs by the solar convective turbulence performed by Musielak \& Rosner (1987, 1988), which were based on the Lighthill theory of sound generation Lighthill (1952) that was modified to account for the magnetic effects, showed no increase in the rates of wave production. Collins (1989a,b) investigated the generation of MAWs in the solar atmosphere by localized sources, and considered the conditions for wave propagation and different mechanisms for wave dissipation. In the above theoretical work, the solar atmosphere was considered to be stratified but isothermal, and the magnetic field to be uniform and either vertical or horizontal.

Similar structures of the solar atmosphere and its magnetic fields were considered in studies of the propagation of
MAWs, performed both analytically and numerically. In analytical work by Thomas (1982) the Wentzel-Kramers-Brillouin (WKB) approximation was used to derive a local dispersion relation from which a cutoff frequency was found for these waves (e.g., Thomas 1983; Campos 1986). Moreover, Nye \& Thomas (1974) investigated MAWs in an idealistic atmosphere. Full analytical solutions to the problem of propagation of MAWs in the isothermal solar atmosphere with uniform and horizontal magnetic field were obtained by Musielak et al. (1989), who demonstrated that in their model there were both standing and propagating waves. To make a distinction between propagating and standing waves, the concept of critical frequency was introduced; the critical frequency is a local quantity resembling a cutoff frequency. Further exploration of the critical and cutoff frequencies was done by Musielak (1990), Musielak et al. (1992), Stark \& Musielak (1993), and in more recent work by Petukhov \& Petukhov (2003) and Roberts (2006).

Numerical work on MAWs was also performed by a number of authors, and in papers by Murawski (1992) and Nakariakov et al. $(1997,1998)$ the nonlinear wave equations were derived for MAWs driven by Alfvén waves. Moreover, Bogdan et al. (2003), Hasan et al. (2005), Khomenko et al. (2008), and Tarr et al. (2017) performed numerical studies of the generation of MAWs in their 2D model, and fast MAWs in random mass density field were discussed by Murawski et al. (2001) to explain the observational value of the wave speed of Moreton and "EIT waves". Magnetoacoustic waves were also extensively studied numerically in the context of vertical loop oscillations (Selwa et al. 2005a, 2006, 2007; Gruszecki et al. 2008, 2009; Wasiljew \& Murawski 2009; Konkol \& Murawski 2010), and the obtained numerical results were found to be in agreement with the observational data of Wang \& Solanki (2004). 
Excitation and attenuation of slow MAWs were explored in solar coronal loops (Selwa et al. 2005b, 2007; Ogrodowczyk et al. 2009; Konkol et al. 2010) and as a mechanism of spicule generation (Murawski \& Zaqarashvili 2010; Kuźma et al. 2017a,b). Analytical and numerical methods for propagation of different MHD waves were reviewed by Murawski (2002). More advanced and comprehensive numerical studies of MHD wave properties in sunspot regions were also extended to higher layers of the solar atmosphere (e.g., Khomenko \& Collados 2015, and references therein). These studies are not restricted to linear waves (e.g., Santamaria et al. 2015, 2016). Khomenko \& Cally (2012) considered geometrical coupling to Alfvén waves, Khomenko \& Collados (2012) discussed partial ionization effects, Felipe et al. $(2010,2011)$ explored the energy transported by the waves, and Felipe et al. (2014) produced forward modeled emission to be compared to observations.

In addition to the extensive analytical and numerical work described above, there have also been a number of reports of observational evidence for the existence of MHD waves in the solar atmosphere. In particular, the existence of Alfvén waves in different regions of the solar atmosphere was confirmed by high-resolution observations performed by the Solar Optical Telescope, the Hinode Solar Observatory, and the Swedish Solar Telescope (e.g., De Pontieu et al. 2007; Cirtain et al. 2007; Jess et al. 2009; Okamoto \& De Pontieu 2011). However, there is some outgoing debate over the real nature of the reported waves (e.g., Erdélyi \& Fedun 2007; Van Doorsselaere et al. 2008). Moreover, Fujimura \& Tsuneta (2009) found MHD waves propagating along solar magnetic flux tubes.

The main aim of this paper is to study the propagation of MAWs in the solar atmosphere numerically and to determine the existence of cutoff periods for these waves and the resulting wave reflection. The considered model of the solar atmosphere is more realistic than that used in previous studies, and the wave behavior is uniquely determined by the gradients of physical parameters, which may lead to partial or total wave reflection. Our studies significantly supplement and extend previous work on driving the solar atmospheric oscillations by acoustic and magnetic flux tube waves, and make theoretical predictions of variations of the cutoff frequencies for MHD waves, which can be verified by future observations. This paper is organized as follows. Our numerical model of the solar atmosphere is presented in Sect. 2; the obtained numerical results are described and discussed in Sect. 3; and conclusions are given in Sect. 4.

\section{Numerical model of the solar atmosphere}

We consider a gravitationally stratified and magnetically permeated solar atmosphere, which is described by the following 2D ideal MHD equations:

$$
\begin{aligned}
& \frac{\partial \varrho}{\partial t}+\nabla \cdot(\varrho \mathbf{V})=0, \\
& \varrho \frac{\partial \mathbf{V}}{\partial t}+\varrho(\mathbf{V} \cdot \nabla) \mathbf{V}=-\nabla p+\frac{1}{\mu}(\nabla \times \mathbf{B}) \times \mathbf{B}+\varrho \mathbf{g}, \\
& \frac{\partial \mathbf{B}}{\partial t}=\nabla \times(\mathbf{V} \times \mathbf{B}), \quad \nabla \cdot \mathbf{B}=0, \\
& \frac{\partial p}{\partial t}+\mathbf{V} \cdot \nabla p=-\gamma p \nabla \cdot \mathbf{V}, \quad p=\frac{k_{\mathrm{B}}}{m} \varrho T,
\end{aligned}
$$

where $\varrho$ is the mass density (see Fig. 1, bottom), $p$ a gas pressure, and $\mathbf{V}=\left[V_{x}, V_{y}, 0\right], \mathbf{B}=\left[B_{x}, B_{y}, 0\right]$, and $\mathbf{g}=[0,-g, 0]$ represent the plasma velocity, the uniform magnetic field, and gravitational acceleration, respectively; we note that the magnitude of $g$ is fixed and equal to $274 \mathrm{~m} \mathrm{~s}^{-2}$. Moreover, $T$ is the plasma temperature, $\gamma=1.4$ is the adiabatic index, $\mu$ is the magnetic permeability of the plasma, $m$ is a particle mass that is specified by a mean molecular weight of 1.24 , and $k_{\mathrm{B}}$ is the Boltzmann's constant. We assume that $z$ is an ignorable coordinate $(\partial / \partial z=0)$. By setting $V_{z}=B_{z}=0$, Alfvén waves are removed from our model.

We assume that the equilibrium plasma is not moving $(\mathbf{V}=\mathbf{0})$ and the solar atmosphere is hydrostatic, that is,

$\varrho_{\mathrm{h}} g=-\frac{\partial p_{\mathrm{h}}}{\partial y}$

such that

$p_{\mathrm{h}}(y)=p_{0} \exp \left[-\int_{y_{\mathrm{r}}}^{y} \frac{\mathrm{d} y^{\prime}}{\Lambda\left(y^{\prime}\right)}\right], \quad \varrho_{\mathrm{h}}(y)=\frac{p_{\mathrm{h}}(y)}{g \Lambda(y)}$,

where the subscript $h$ corresponds to a hydrostatic quantity, $y_{\mathrm{r}}=10 \mathrm{Mm}$ is the reference level, $p_{0}$ is the gas pressure at this level and

$\Lambda(y)=\frac{k_{\mathrm{B}} T_{\mathrm{h}}(y)}{m g}$,

which is the pressure scale-height that depends on the plasma temperature $T_{\mathrm{h}}(y)$, the latter being taken from the semi-empirical model of Avrett \& Loeser (2008); see Fig. 1 (top).

The hydrostatic atmosphere is stratified in the vertical or $y$ direction, however it remains homogeneous in the horizontal direction. It must be pointed out that within the framework of this model the solar photosphere occupies the region given by $0<y<0.5 \mathrm{Mm}$. At $y \approx 0.6 \mathrm{Mm}$ the hydrostatic temperature $T_{\mathrm{h}}(y)$ reaches its minimum of about $4300 \mathrm{~K}$ and $T_{\mathrm{h}}(y)$ slowly increases with height in the chromosphere up to the transition region, which is located at $y \approx 2.1 \mathrm{Mm}$. Across this region $T_{\mathrm{h}}(y)$ experiences a rapid growth to the coronal value of about $0.8 \mathrm{MK}$ at $y=10 \mathrm{Mm}$, and higher up $T_{\mathrm{h}}(y)$ reaches a value of about $2.5 \mathrm{MK}$.

We consider two different structures of magnetic field: the uniform horizontal, $\mathbf{B}=\left[B_{0}, 0,0\right]$, and vertical, $\mathbf{B}=\left[0, B_{0}, 0\right]$, magnetic fields. We fix the magnitude of the magnetic field at $B_{0} \approx 11.4 \mathrm{G}$ which is a typical value for a quiet solar region (e.g., Morgan \& Taroyan 2017).

\section{Numerical simulations}

We solve the MHD Eqs. (1)-(4) numerically using the FLASH code (Lee \& Deane 2009; Lee 2013), and perform our numerical simulations with an adaptation of the van Leer slope limiter and the HLLD approximate Riemann solver (Toro 2009). We set the simulation box as $(-2,2) \mathrm{Mm} \times(0,80) \mathrm{Mm}$ and cover it by a static, nonuniform grid with a minimum (maximum) level of refinement set to 1 (7). We note that grid refinement leads to smaller cells. The first level of refinement corresponds to the basic blocks of their size of $4 \mathrm{Mm} \times 4 \mathrm{Mm}$ with each block consisting of $8 \times 8=64$ identical cells. As a result, a coarse cell is $0.5 \mathrm{Mm}$ wide along each direction. The seventh level of refinement leads to cells of $7.8 \mathrm{~km}$ wide below the level $y=2.5 \mathrm{Mm}$. A coarse grid is used to cover the higher coronal region. The top boundary of the simulation box is located at $y=80 \mathrm{Mm}$ and is chosen to be high enough to minimize wave reflections from this boundary by numerically diffusing the incoming signal. At all four boundary conditions we set all plasma quantities to their equilibrium values. The background state of the atmosphere is constructed with the help of Eqs. (5)-(7) with the uniform magnetic field. 

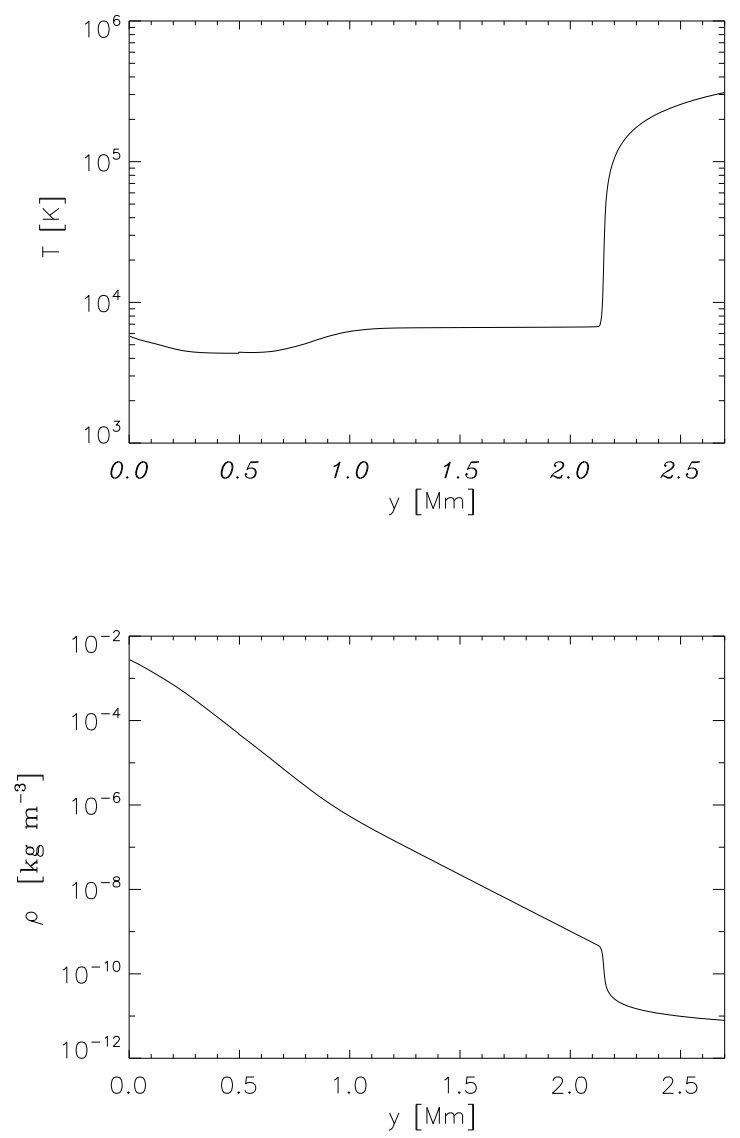

Fig. 1. Vertical profiles of equilibrium temperature (top panel) and mass density (bottom panel) profiles.

Additionally, a periodic driver in the $y$-component of the velocity is introduced at the bottom $(y=0)$ boundary in the form of the horizontally oriented wave front, which is given by

$V_{y}(x, y, t)=V_{0} \exp \left(-\frac{y^{2}}{w^{2}}\right) \sin \left(\frac{2 \pi t}{P_{d}}\right)$,

where $V_{0}$ is the amplitude of the driver, $w$ is its width, and $P_{d}$ represents its period. We take $V_{0}=0.025 \mathrm{~km} \mathrm{~s}^{-1}$ and $w=100 \mathrm{~km}$. Since the medium is magnetized and the driver is homogeneous along the horizontal direction, internal gravity waves are not excited as they are not able to propagate along the direction of the solar gravity. As a result, the driver excites fast and slow MAWs only, which become affected by the gravity while propagating through the solar chromosphere towards the corona.

\subsection{Magnetoacoustic waves in the model}

In this part of the paper, we consider MAWs in a 1D model in which all perturbations are invariant along the horizontal direction, and the waves are only able to propagate vertically. From the physical point of view, fast and slow MAWs exist because of perturbations of the background gas and magnetic pressures. For linear waves, the perturbations are small compressions and rarefactions of gas and magnetic pressures, which add up together for fast MAWs and subtract from each other for slow MAWs. As a result, both waves propagate with distinct characteristic velocities that are typically obtained from the global (e.g., Osterbrock 1961) or local (e.g., Thomas 1983) dispersion relations. The
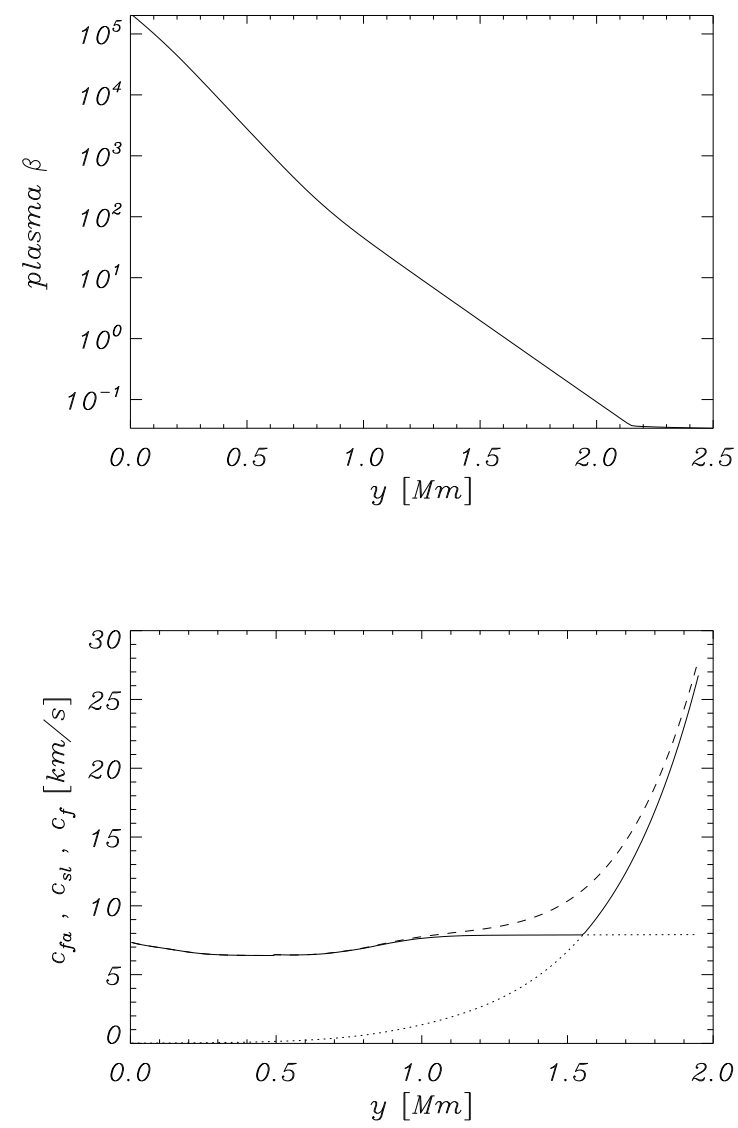

Fig. 2. The plasma $\beta$ (top panel) and the fast $c_{\mathrm{fa}}$ (solid line) and slow $c_{\mathrm{s}}$ (dotted line) characteristic speeds for MAWs in the model with the uniform vertical magnetic field, and the fast $c_{\mathrm{f}}$ (dashed line) speed (bottom panel) in the uniform horizontal magnetic field case.

characteristic velocities for fast and slow MAWs are given by

$c_{\mathrm{fa}, \mathrm{sl}}^{2}=\frac{1}{2} c_{f}^{2} \pm \frac{1}{2} \sqrt{c_{f}^{4}-4 c_{\mathrm{s}}^{2} c_{\mathrm{A}}^{2} \cos ^{2} \theta}$,

where $c_{\mathrm{fa}}$ (positive sign) and $c_{\mathrm{sl}}$ (negative sign) are the phase velocities of fast and slow MAWs, respectively. In addition, $c_{f}^{2}(y)=c_{\mathrm{s}}^{2}(y)+c_{\mathrm{A}}^{2}(y), c_{\mathrm{s}}^{2}=\gamma p_{\mathrm{h}} / \varrho_{\mathrm{h}}$, and $c_{\mathrm{A}}^{2}=B_{0}^{2} /\left(\mu \varrho_{\mathrm{h}}\right)$ are respectively the squared fast, sound, and Alfvén speeds, and $\theta$ is the angle between the direction of wave propagation and the direction of a uniform magnetic field.

We use the definitions of $c_{\mathrm{fa}}$ and $c_{\mathrm{sl}}$ to determine the characteristic phase velocities for MAWs propagating in our model. Since the sound speed follows the temperature variation in the model and Alfvén velocity depends on the mass density distribution or stratification in the model, then in our nonisothermal and stratified solar atmosphere both $c_{\mathrm{s}}$ and $c_{\mathrm{A}}$ vary with height, and as a result, $c_{\mathrm{fa}}$ and $c_{\mathrm{sl}}$ are also functions of the atmospheric height. For the vertical field, $\theta=0$, and in the case of $c_{\mathrm{s}}>c_{\mathrm{A}}$, fast and slow MAWs propagate with $c_{\mathrm{fa}}=c_{\mathrm{s}}$ and $c_{\mathrm{sl}}=c_{\mathrm{A}}$, and these waves become decoupled and their physical properties are different; the fast waves are longitudinal with the gas pressure perturbations responsible for their existence However, the slow waves are magnetic in nature and the magnetic field perturbations are responsible for their existence. For $c_{\mathrm{A}}>c_{\mathrm{s}}$, the wave characteristic velocity changes to $c_{\mathrm{fa}}=c_{\mathrm{A}}$ and $c_{\mathrm{sl}}=c_{\mathrm{s}}$, and the physical picture underlying these waves is reversed (Musielak \& Rosner 1987).

Now, for the horizontal magnetic field, $\theta=90^{\circ}$ and only fast MAWs exist, and their phase velocity is $c_{\mathrm{f}}$ again; the waves are 
either acoustic-like if $c_{\mathrm{s}}>c_{\mathrm{A}}$ or magnetic-like in the opposite case. There are no slow MAWs propagating across the magnetic field lines for the horizontal magnetic field. Analytical solutions for an isothermal atmosphere were obtained by Thomas (1983) and Musielak et al. (1989), who demonstrated that Thomas' solutions were valid only for the downward wave propagation, and derived the solutions for the upward wave propagation.

We use the above considerations in our interpretation and discussion of the obtained numerical results. It is also convenient to introduce the plasma $\beta$, defined as the ratio of a gas pressure, $p_{\mathrm{h}}(y)$, and magnetic pressure, $B_{0}^{2} /(2 \mu)$, or equivalently as the ratio of $2 c_{\mathrm{s}}^{2} /\left(\gamma c_{\mathrm{A}}^{2}\right)$. The plasma $\beta$ in the solar atmosphere is plotted in Fig. 2 (top), which shows that in our atmospheric model the plasma $\beta>1$ for $y<1.6 \mathrm{Mm}$, and $\beta<1$ in the upper layers. As a result, we infer that in the case of the vertical magnetic field, slow and fast MAWs are coupled in the low atmospheric layers, while for $y>1.6 \mathrm{Mm}$ the coupling is smaller and these waves are easily distinguishable with perturbations in $V_{y}\left(V_{x}\right)$ corresponding to slow (fast) MAWs.

In Fig. 2 (bottom), the characteristic wave speeds for MAWs are presented, and it is seen that all speeds are continuous and smooth functions of the atmospheric height. The main reason is the fact that the speeds are determined by the smooth distribution of physical parameters in the atmosphere. The effects of these characteristic speeds on the propagation of MAWs is now discussed.

\subsection{Cutoff frequencies for magnetoacoustic waves}

The propagation of MAWs in the solar atmosphere is affected by cutoff periods for these waves. Among different attempts to determine the cutoff for MAWs in the isothermal solar atmosphere with the uniform horizontal and vertical magnetic fields (e.g., Thomas 1983; Campos 1986; Musielak 1990; Stark \& Musielak 1993; Petukhov \& Petukhov 2003; Roberts 2006), we select the local cutoff period for MAWs obtained by Stark \& Musielak (1993), which is given by

$P_{k}(y)=\frac{4 \pi \Lambda}{\sqrt{c_{\mathrm{s}}^{2}+\frac{3}{4}\left(\frac{c_{\mathrm{A}}}{c_{\mathrm{f}}}\right)^{2} c_{\mathrm{A}}^{2}}}$,

for the horizontal magnetic field, and the local cutoff derived for the vertical magnetic field by Roberts (2006) and defined as

$P_{\mathrm{m}}(y)=2 \pi / \Omega_{\mathrm{m}}$,

where

$\Omega_{\mathrm{m}}^{2}(y)=c_{t}^{2}\left\{\frac{1}{4 \Lambda^{2}}\left(\frac{c_{t}}{c_{\mathrm{s}}}\right)^{4}-\frac{1}{2} \gamma g\left(\frac{c_{t}^{2}}{c_{\mathrm{s}}^{4}}\right)^{\prime}+\frac{1}{c_{\mathrm{A}}^{2}}\left(\omega_{g}^{2}+\frac{g}{\Lambda} \frac{c_{t}^{2}}{c_{\mathrm{s}}^{2}}\right)\right\}$,

with $\omega_{g}^{2}$ denoting the squared buoyancy or Brunt-Väisälä frequency given by

$\omega_{g}^{2}=-g\left(\frac{g}{c_{s}^{2}}+\frac{\varrho^{\prime}}{\varrho}\right)$

with $c_{t}=c_{\mathrm{s}} c_{\mathrm{A}} / c_{f}$ being the "cusp" (or tube) speed and ' means $\mathrm{d} / \mathrm{d} y$.

The basic idea that underlines the derivation of these cutoff frequencies goes back to Lamb (1909, 1910, 1945) who obtained the cutoff frequency for acoustic waves in an isothermal atmosphere, and found the global (the same in the entire atmosphere) acoustic cutoff frequency. The original
Lamb derivation involves linearizing the standard ideal hydrodynamic equations and obtaining the wave equation written in its standard form, in which any term with the first derivative is removed. The resulting wave equation (Lamb, or known in the literature as Klein-Gordon equation; see Rae \& Roberts 1982; Musielak et al. 1992) directly displays a term that is typically interpreted as a cutoff (or critical) frequency (or associated with such a period).

The same procedure was used by Stark \& Musielak (1993) and Roberts (2006), who linearized the MHD equations, removed the resulting terms with the first-order derivatives, and cast the wave equations in their standard forms. The expressions for cutoff frequencies given above were originally obtained by these authors from their derived Lamb (or Klein-Gordon) wave equations, who showed that in the case of a horizontal magnetic field, the only waves to propagate are those with period $P$ shorter than the cutoff period $P<P_{k}$, and otherwise the waves become evanescent. The main difference between Lamb's acoustic cutoff and the above cutoffs for MAWs is the fact that the latter are local quantities that vary with atmospheric height.

The analytically obtained cutoffs given above are compared to those found numerically in the following sections.

\subsection{The horizontal magnetic field case}

In the uniform horizontal magnetic field, the periodic driver given by Eq. (8) excites fast MAWs, which are the only waves allowed to travel across the magnetic field lines (see Sect. 3.1).

Figure 3 illustrates time signatures (upper parts of panels) collected at $y=0.9 \mathrm{Mm}$, which corresponds to the low chromosphere (top panels), and $y=1.5 \mathrm{Mm}$, lying about $600 \mathrm{~km}$ below the transition region (bottom panels). The corresponding Fourier periodograms obtained for the driving wave period $P_{d}=300 \mathrm{~s}$ are displayed in the lower parts. At the atmospheric height $y=0.9 \mathrm{Mm}$, the main wave period corresponding to the maximum power is equal to the driving period that is $P=P_{\mathrm{d}}=300 \mathrm{~s}$, and in addition, the second main period $P \approx 210 \mathrm{~s}$ becomes excited. Higher up in the atmosphere, the second main period remains unchanged. However, the main first period is significantly reduced, which also results in the maximum power being shifted to $P \approx 210$ s waves. This clearly shows that MAWs with their wave periods of $P=300 \mathrm{~s}$ are evanescent and that their amplitude decays with height $y$.

We note that the amplitude of the signal collected at $y=$ 1.5 Mm is larger than the amplitude at $y=0.9 \mathrm{Mm}$ (Fig. 3). The signal at $y=0.9 \mathrm{Mm}$ (top panels) is a wave package that contains two main wave periods, namely $P \approx 210 \mathrm{~s}$ and $P=300 \mathrm{~s}$. The latter signal is evanescent and its amplitude decays with height. However, the oscillations of $P \approx 210$ s grow in their amplitude with height. This growth is a result of the equilibrium mass density fall off with $y$ (Fig. 1, bottom), owing to the presence of gravity; see Eq. (6). As a consequence of this growth the amplitude of the whole package of waves is higher at $y=1.5 \mathrm{Mm}$ than at $y=0.9 \mathrm{Mm}$ (Fig. 3).

We found that for the driving period, $P_{d}=200 \mathrm{~s}$, which is lower than $P_{k}(y)$ given by Eq. (10) for most of the atmosphere (the only exception are the layers at the transition region). Furthermore, the fast MAWs propagate freely from the photosphere to the corona with the unchanged first main period and the second main period of about $100 \mathrm{~s}$ (Fig. 4). The second main period is half of the driving period. This period corresponds to the second harmonic (e.g., Chae et al. 2018) that is driven as a result of nonlinear effects (as seen in the sawtooth profiles illustrated in Fig. 4, bottom). We also note that the amplitudes of 

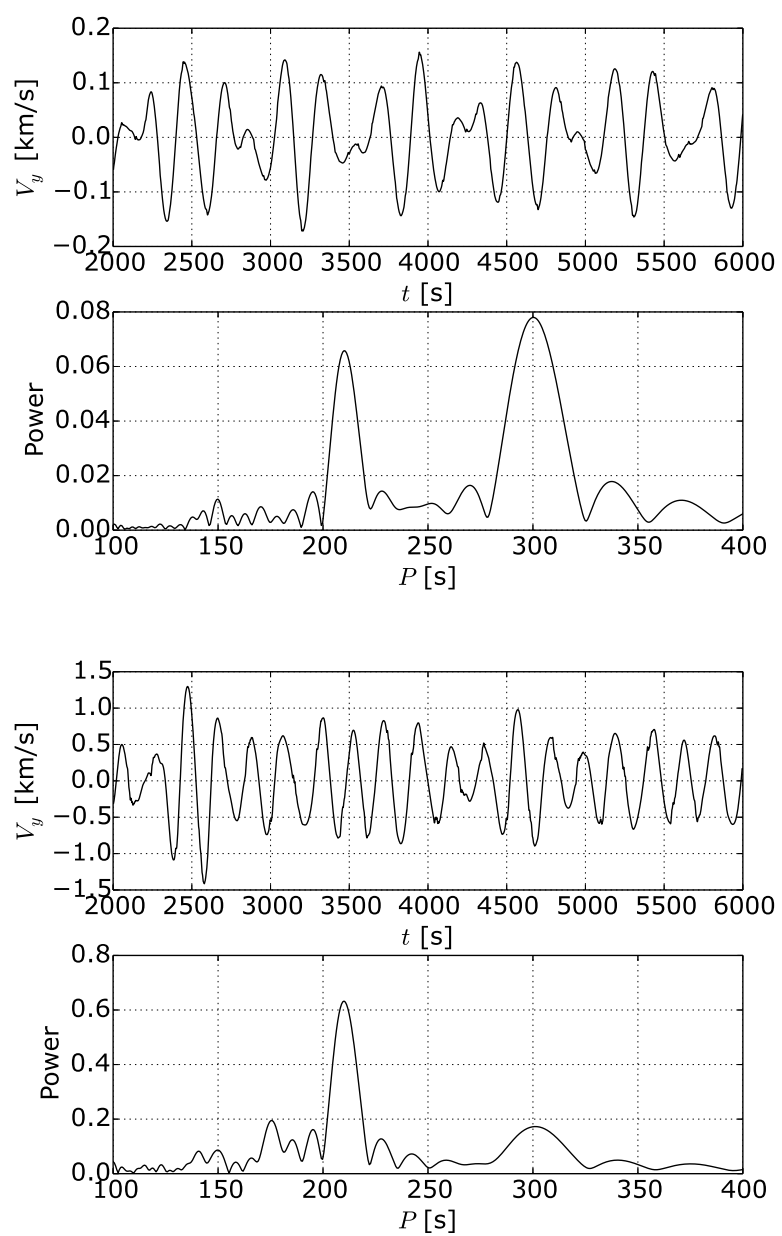

Fig. 3. Time signatures (upper parts) and their Fourier periodograms (lower parts) of $V_{y}(y)$ for $y=0.9 \mathrm{Mm}$ (top panel) and $y=1.5 \mathrm{Mm}$ (bottom panel) for the uniform horizontal magnetic field and $P_{\mathrm{d}}=$ $300 \mathrm{~s}$.

the signals collected at the detection points are higher in the case of $P_{\mathrm{d}}=200 \mathrm{~s}$ than for $P_{\mathrm{d}}=300 \mathrm{~s}$, which can also serve as evidence of wave evanescence in the latter case. The evanescence of waves, both in the case of $P_{\mathrm{d}}=300 \mathrm{~s}$ and $P_{\mathrm{d}}=200 \mathrm{~s}$, in the narrow region where $P_{k}(y)<P_{\mathrm{d}}$ is so insignificant that we do not observe any amplitude decrease there. We note that wave tunneling is the phenomenon associated with the passage of evanescent waves as they move into heights where their period is forbidden. Hence, the waves tunnel into the low corona through the forbidden zone around the transition region.

Figure 5 displays the two main periods of $V_{y}$ for the driving wave period $P_{\mathrm{d}}=300 \mathrm{~s}$ (top) and $P_{\mathrm{d}}=200 \mathrm{~s}$ (bottom). The analytically obtained cutoff period $P_{k}$ is depicted by solid lines and serves for comparison purposes. We note that $200 \mathrm{~s}<$ $P_{k}<255 \mathrm{~s}$ for $0<y<1.6 \mathrm{Mm}$ (Fig. 5, solid line). Higher up, $P_{k}$ declines, attaining its local minimum of $P_{k} \approx 50 \mathrm{~s}$ at $y \approx 2.1 \mathrm{Mm}$ and then it grows with $y$, reaching a value of $P_{k} \approx 330 \mathrm{~s}$ at $y=3.0 \mathrm{Mm}$. According to the analytical prediction by Stark \& Musielak (1993), MAWs with the wave period $P>P_{k}$ are not able to propagate freely into higher atmospheric regions. Instead, the waves experience reflection due to gradients of the characteristic wave speed $c_{\mathrm{f}}$, which are most pronounced from the level of minimum temperature (Fig. 2, bottom). The resulting wave reflection is obviously more important for higherperiod waves as short-period waves are not affected by the reflection and therefore they propagate essentially freely as in a locally
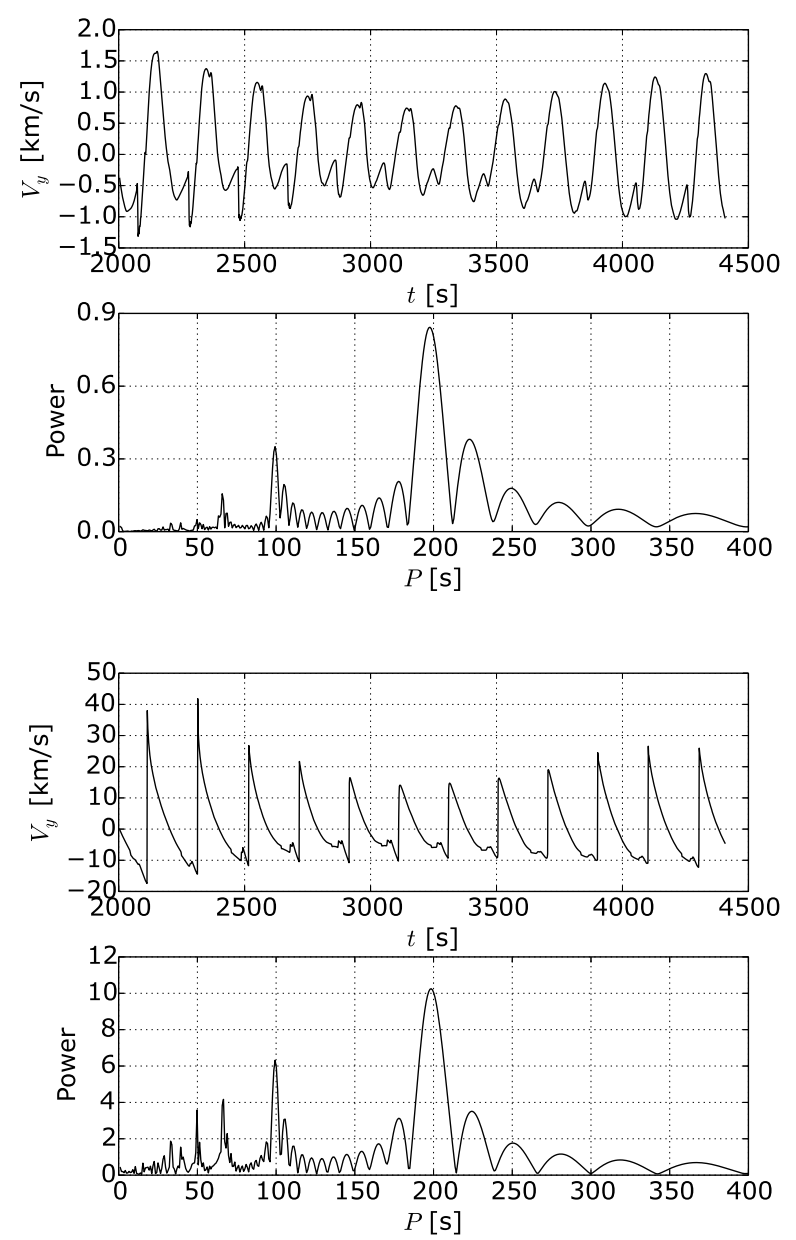

Fig. 4. Time signatures (upper parts) and their Fourier periodograms (lower parts) of $V_{y}(y)$ for $y=1.0 \mathrm{Mm}$ (top panels) and $y=2.0 \mathrm{Mm}$ (bottom panels) for the uniform horizontal magnetic field case and $P_{\mathrm{d}}=$ $200 \mathrm{~s}$.

homogeneous medium. The waves with $P_{d}=300 \mathrm{~s}$ tunnel their energy up to the atmospheric height of $y=1 \mathrm{Mm}$, and transform their energy to the waves with $P \approx 210$ s. These waves reach the level of $y \approx 0.9 \mathrm{Mm}$ for $P_{\mathrm{d}}=300 \mathrm{~s}$ (Fig. 5, top).

Figure 6 schematically illustrates the scenario of wave excitation. The waves of $P<P_{\max }=230$ s, which we call the maximal period, $P_{\max }$, propagate freely into the solar corona, while the waves with higher wave periods are evanescent, and transfer their energy, in the region given by $y>0.6 \mathrm{Mm}$, into the waves of $P=210 \mathrm{~s}$, which at $y \approx 1 \mathrm{Mm}$ and higher up become dominant.

Let us now explain our numerical results. As seen in Fig. 5, the cutoff $P_{k}$ shows two depressions: one quite broad depression around the point $y \simeq 0.5 \mathrm{Mm}$ and the other in the area surrounding $y=2.1 \mathrm{Mm}$, where waves with $P>P_{k}$ cannot propagate freely. In this case the region of free propagation is shifted to lower heights, mainly between approximately $0.8 \mathrm{Mm}$ and $1.9 \mathrm{Mm}$. These characteristic points are well seen in Fig. 7, which shows the vertical component of velocity versus the height at two different moments of time, and in two opposite phases, and at the maximum absolute value of velocity. At $t=5000 \mathrm{~s}$ the profile of $V_{y}$ rapidly grows in the region between 0.8 and $1.9 \mathrm{Mm}$ and abruptly stops changing for $y>1.9 \mathrm{Mm}$ akin to a standing wave pattern. Such a configuration reoccurs with the period of about $210 \mathrm{~s}$. The standing waves set up in this region with their wave period $P \approx 2 L / \bar{c}_{\mathrm{f}}=206 \mathrm{~s}$, which is close to the excited wave period $P=210 \mathrm{~s}$. Here, the spatially averaged 

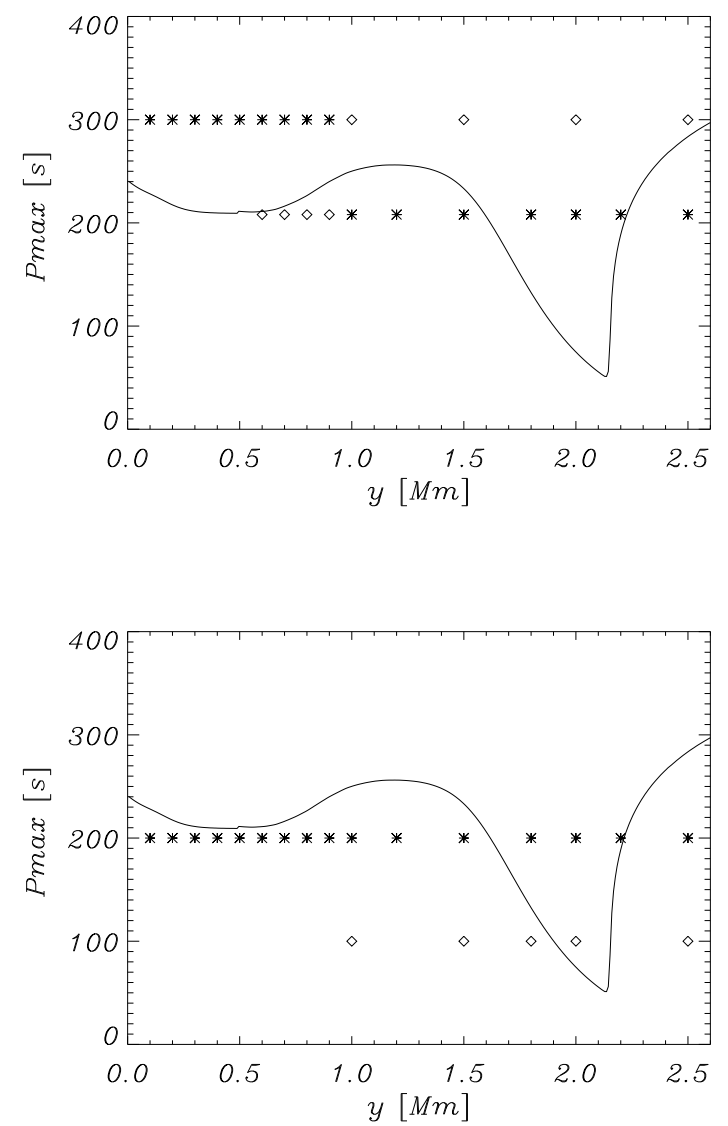

Fig. 5. First (asterisks) and second (diamonds) main periods of $V_{y}$ vs. height $y$ in the horizontal magnetic field case for $P_{\mathrm{d}}=300 \mathrm{~s}$ (top panel) and $P_{\mathrm{d}}=200 \mathrm{~s}$ (bottom panel). The cutoff wave period $P_{k}$ given by Eq. (10) is depicted by solid lines.

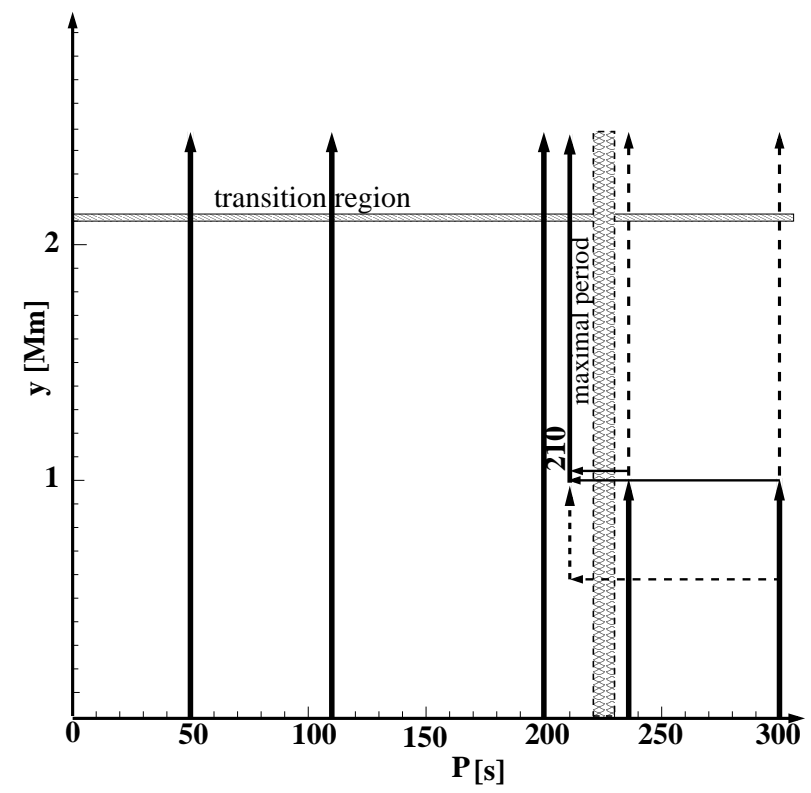

Fig. 6. Scheme of the excited waves for the horizontal magnetic case.

fast speed $\bar{c}_{\mathrm{f}} \approx 10.7 \mathrm{~km} \mathrm{~s}^{-1}$. The time difference between the opposite phases displayed in Fig. 7 is $100 \mathrm{~s}$ which is close to half of the period of the excited standing wave.

The main excited wave period depends on the value of the horizontal magnetic field (Fig. 8). This period depends on

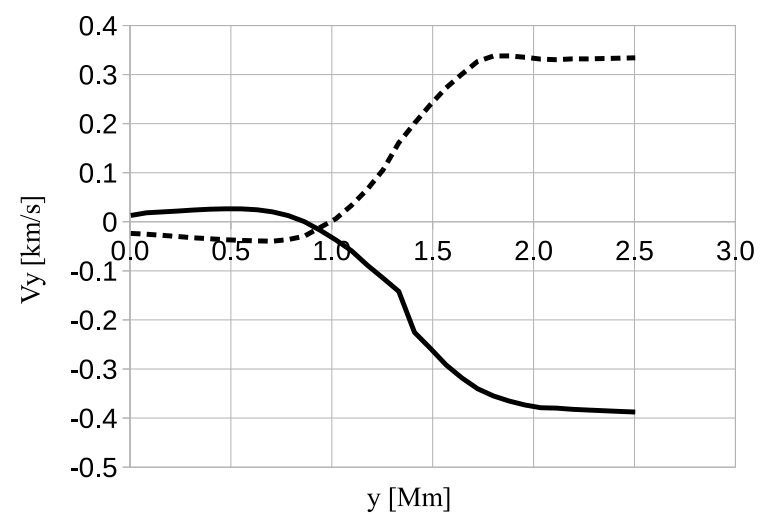

Fig. 7. Spatial profiles of $V_{y}(y, t)$ at $t=4900 \mathrm{~s}$ (solid line) and $t=5000 \mathrm{~s}$ (dash line) in the horizontal magnetic case and $P_{\mathrm{d}}=300 \mathrm{~s}$.

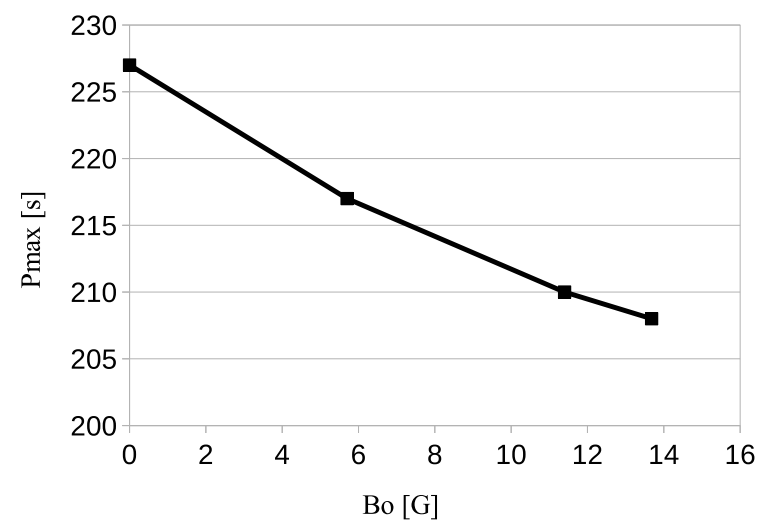

Fig. 8. Main wave periods of excited waves vs. the magnitude of horizontal magnetic field for $P_{\mathrm{d}}=300 \mathrm{~s}$.

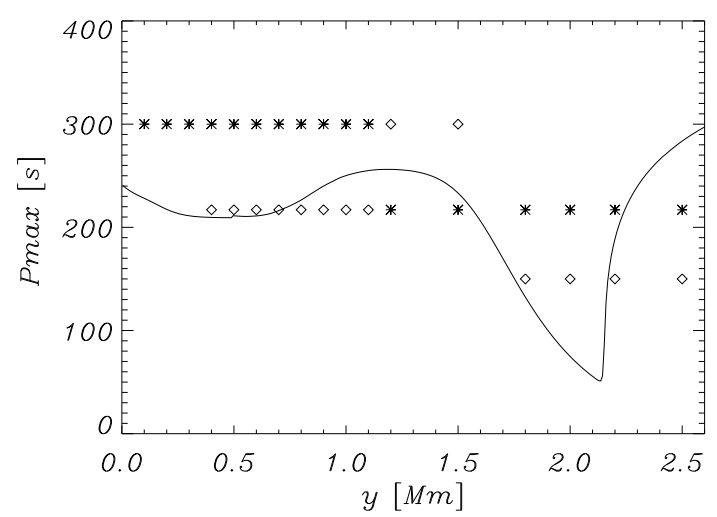

Fig. 9. First (asterisks) and second (diamonds) main periods of $V_{y}$ vs. height $y$ in the horizontal magnetic field case for $P_{\mathrm{d}}=300 \mathrm{~s}$ for a weaker horizontal magnetic field with $B_{0}=5.6 \mathrm{G}$. The cutoff wave period $P_{k}$ given by Eq. (10) is depicted by solid lines.

the velocity of the fast MAWs, which in turn depends on the magnetic field. The greater the magnetic field strength, the greater the velocity $\bar{c}_{\mathrm{f}}$ and the smaller the period of the waves.

In Fig. 9 two main periods, $V_{y}$ and the cutoff period $P_{k}(y)$, are depicted in the case of $P_{\mathrm{d}}=300 \mathrm{~s}$ and weak horizontal magnetic field $\left(B_{0}=5.6 \mathrm{G}\right)$. Comparing Figs. 9 and 5 (top panel), we infer that for the weak magnetic field $P=300 \mathrm{~s}$ is dominant up to $y \approx 1.1 \mathrm{Mm}$ (Fig. 9), which is about $200 \mathrm{~km}$ higher than in the case of the twice stronger magnetic field (Fig. 5, top). 

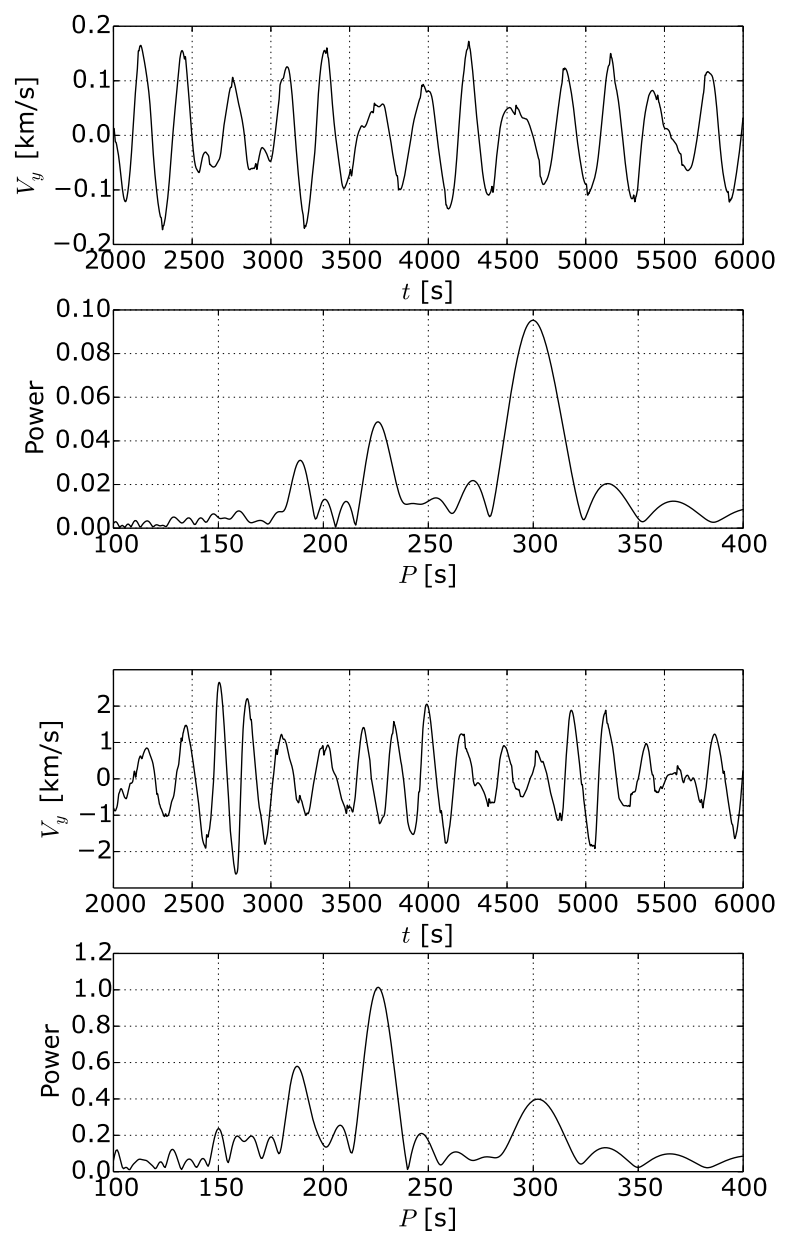

Fig. 10. Time signatures (upper parts) and their Fourier periodograms (lower parts) of $V_{y}(y)$ for $y=1.0 \mathrm{Mm}$ (top panels) and $y=2.0 \mathrm{Mm}$ (bottom panels) in the uniform vertical magnetic field case and $P_{\mathrm{d}}=$ $300 \mathrm{~s}$.

\subsection{The vertical magnetic field case}

We consider now the case of the vertical magnetic field and the periodic driver given by Eq. (8). As in low atmospheric regions, MAWs propagate in the medium of high plasma $\beta$ (Fig. 2, top), the fast and slow MAWs have similar properties. However, higher up in the atmosphere, the wave signal reaches the altitudes of low values of $\beta$ and perturbations in $V_{y}$ are associated with slow MAWs.

Figure 10 illustrates time signatures and the associated Fourier spectra evaluated at the level $y=1.0 \mathrm{Mm}$ corresponding to the low chromosphere (top panels), and $y=2.0 \mathrm{Mm}$, located about $100 \mathrm{~km}$ below the transition region (bottom panels). We note that at $y=1.0 \mathrm{Mm}$ the first main period of excited waves corresponds to $300 \mathrm{~s}$ and the second main period is located at $P=227 \mathrm{~s}$ (top panels). This is completely understood as at the detection height the plasma $\beta$ is very high (Fig. 2), and as a result, the slow and fast MAWs propagate upward coupled together with essentially the same speed (Fig. 2, bottom). Just $100 \mathrm{~km}$ below the transition region the period located at $P=300 \mathrm{~s}$ evanesces and the period located at $P=227 \mathrm{~s}$ becomes the first main period. The new period at $P=188 \mathrm{~s}$ is energized, itself becoming the second main period.

We conclude that the Fourier spectra of excited waves are similar in horizontal (Fig. 3) and vertical (Fig. 10) magnetic field cases with the only main difference being that for the horizontal
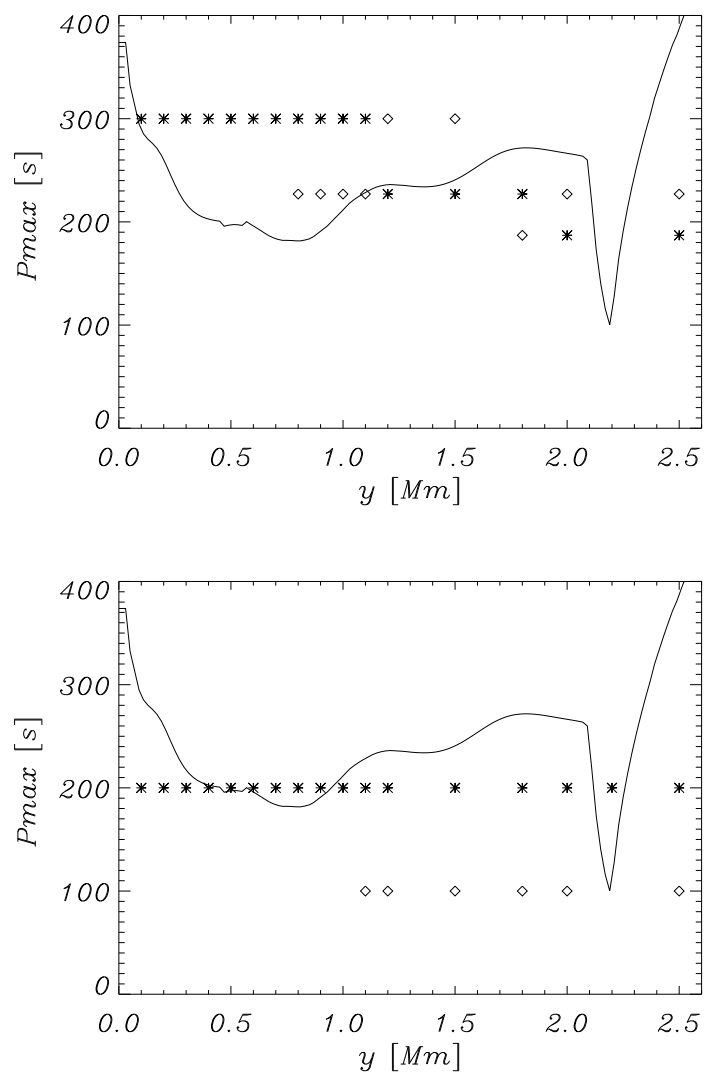

Fig. 11. Main periods of $V_{y}$ (asterisks) of magnetoacoustic waves vs. height $y$ in the vertical magnetic field case and $P_{\mathrm{d}}=300 \mathrm{~s}$ (top panel) and $P_{\mathrm{d}}=200 \mathrm{~s}$ (bottom panel). The cutoff wave period $P_{m}$ is depicted by a solid line.

magnetic field the waves of the maximal period, $P_{\max }=210 \mathrm{~s}$, are excited, while for the vertical magnetic field, $P_{\max }=227 \mathrm{~s}$. Parametric studies were performed to verify that, for different values of $P_{\mathrm{d}}$, the waves of periods greater than $255 \mathrm{~s}$ decline with height. A sample result is presented in Fig. 10. However, the waves of periods smaller than $255 \mathrm{~s}$ propagate freely upwards reaching the transition region and the corona.

Figure 11 displays the two main periods of $V_{y}$ for the driving wave period $P_{\mathrm{d}}=300 \mathrm{~s}$ (top) and $P_{\mathrm{d}}=200 \mathrm{~s}$ (bottom). The analytically obtained cutoff period $P_{m}$ (see Sect. 2.3) is depicted by solid lines and can be used for comparison purposes. According to the analytical theory of Roberts (2006) waves with wave periods $P>P_{m}$ are not able to propagate freely into higher atmospheric regions. However, the waves reach the level of $y \approx 1.2 \mathrm{Mm}$ if $P_{\mathrm{d}}=300 \mathrm{~s}$ (top). We note that in the case of $P_{\mathrm{d}}=300 \mathrm{~s}$ the first and second main detected wave periods swap over at the level $y=1.2 \mathrm{Mm}$ at which the main wave period corresponds to $P \approx 227 \mathrm{~s}$ (top). As for the waves with $P=300 \mathrm{~s}$ their $P$ is lower than the local value of $P_{m}(y)$ and these waves can propagate freely up to $y \approx 0.6 \mathrm{Mm}$. Higher up $P_{d}>P_{m}(y)$ and these waves become evanescent; they tunnel their energy at the level of $y=1.2 \mathrm{Mm}$ and transform themselves into waves with $P_{\max } \approx 227 \mathrm{~s}$. For the driver with its period $P_{\mathrm{d}}=200 \mathrm{~s}$ the excited waves propagate freely into the corona (Fig. 11, bottom).

Figure 12 summarizes the scenario of wave propagation schematically. This scenario of the vertical propagation MAWs is qualitatively similar to the case of the horizontal magnetic field. The only difference is that in this case the maximal period $P_{\max }$ is about $255 \mathrm{~s}$ and the excited wave period for $P>P_{\mathrm{d}}$ is $227 \mathrm{~s}$. The latter can be explained along the lines done for the case of 


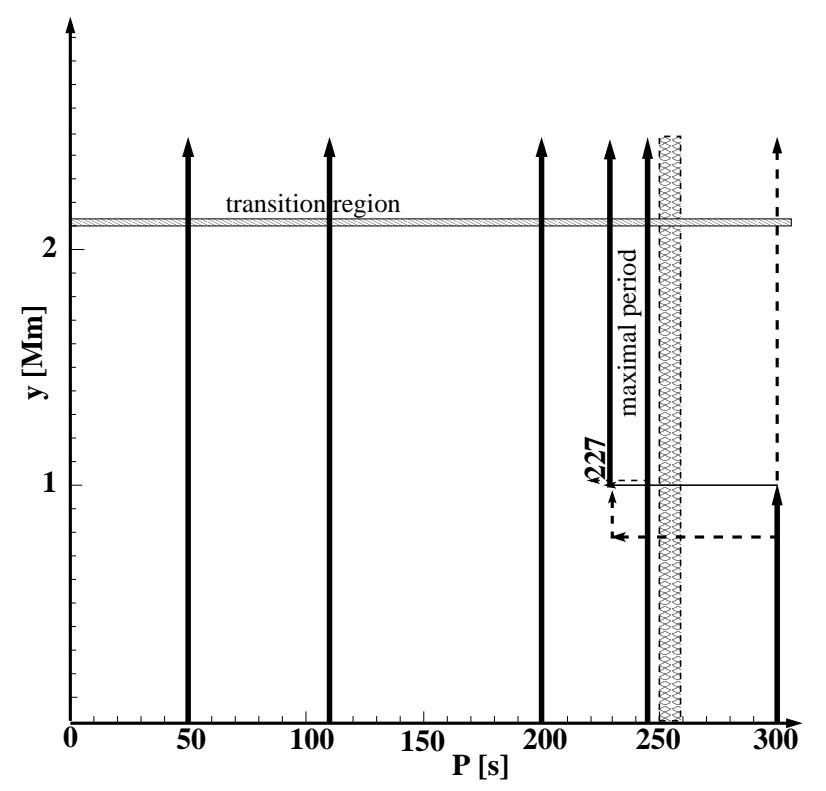

Fig. 12. Scheme of the excited waves for the vertical magnetic field case.

the horizontal magnetic field, mainly by the existence of standing waves between $y \approx 1.1 \mathrm{Mm}$ and $y \approx 2.1 \mathrm{Mm}$. The profile of the standing wave in the vertical magnetic case is shown in Fig. 13.

\subsection{Comparison between our results and the literature}

The outer solar atmosphere is known to oscillate with a period that is different from that of the photosphere. While the latter is dominated by 5 min oscillations, the main oscillations of the solar chromosphere are near $3 \mathrm{~min}$. From a theoretical point of view, there are fundamental differences in the origin of these oscillations. The solar 5 min oscillations are caused by the so-called cavity modes, which are essentially acoustic waves trapped inside the Sun (e.g., Ulrich 1970; Leibacher \& Stein 1971). However, the solar 3 min oscillations are likely to be a response of the solar chromosphere to propagating acoustic waves, which not only heat the chromosphere (e.g., Ulmschneider et al. 2001) but also excite free atmospheric oscillations at the acoustic cutoff frequency (Fleck \& Schmitz 1991).

Extensive analytical (Fleck \& Schmitz 1993; Kalkofen et al. 1994; Schmitz \& Fleck 1995; Sutmann et al. 1998) and numerical (Sutmann \& Ulmschneider 1995a,b) studies were performed of the behavior of acoustic waves in the solar atmosphere. The main obtained results can be summarized as follows. The free atmospheric oscillations are always present, independent of the form of the initial disturbance that caused them, and they decay in time as $t^{-3 / 2}$ if the periods of the driving waves are above the acoustic cutoff period. Obviously, there are also the so-called forced atmospheric oscillations, which represent either propagating or evanescent acoustic waves, and do not decay in time if the wave source drives them continuously. Moreover, in the special case when the wave frequency is exactly equal to the acoustic cutoff frequency, the free and forced oscillations are the same and they do not decay in time.

Observations of $\mathrm{Ca}$ II $\mathrm{H}$ and $\mathrm{K}$ lines, the $\mathrm{Ca}$ II infrared triplet lines, and the $\mathrm{H} \alpha$ line show that the 3 min chromospheric oscillations range from 2 to $5 \mathrm{~min}$ inside nonmagnetic or weak magnetic regions (supergranulation cells). However, in magnetic regions located at the boundaries of supergranules (the magnetic network), the oscillations range from 6 to $15 \mathrm{~min}$ (e.g., Dame

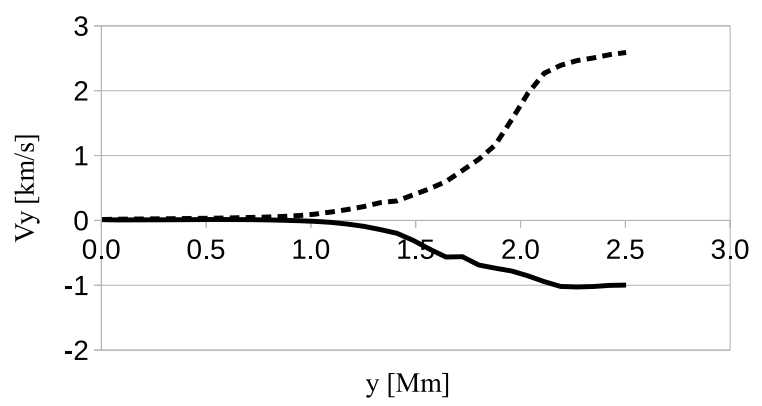

Fig. 13. Spatial profiles of $V_{y}(y, t)$ at $t=4800 \mathrm{~s}$ (solid line) and $t=$ $4900 \mathrm{~s}$ (dash line) in the vertical magnetic field case and $P_{\mathrm{d}}=300 \mathrm{~s}$.

1983; Deubner 1991; McAteer et al. 2002, 2003; Tritschler et al. 2005). Previously obtained theoretical results seem to indicate that the chromospheric oscillations are driven by freely propagating acoustic waves (e.g., Fleck \& Schmitz 1991; Kalkofen 1997; Sutmann et al. 1998) in magnetic-free regions, and by magnetic flux tube waves (e.g., Bogdan et al. 2003; Hasan et al. 2005). The excitation of oscillations in magnetic regions of the solar atmosphere was investigated by Musielak \& Ulmschneider (2003a,b), who considered waves in thin magnetic flux tubes embedded in the solar atmosphere.

In the above studies, the origin of the chromospheric oscillations was identified with freely propagating acoustic or magnetic (flux tube) waves. However, there were also reports on formation of a chromospheric cavity by partial reflection of acoustic waves between a photospheric temperature drop and the steep temperature gradient in the solar transition region (e.g., Schmitz \& Fleck 1992). The formation of such a cavity would require a well defined lower boundary that would cause the waves to be physically reflected backward into the cavity. Since there is no such lower boundary in our model, the concept of chromospheric cavity is not considered in this paper.

Numerical simulations of MAWs performed here to determine their role in driving the chromospheric oscillations significantly supplement and extend previous studies described above. We found the cutoffs and their variation with height, and compared these numerical cutoffs to those obtained analytically. It is also noteworthy that the chromospheric oscillations predicted by our theoretical studies are more consistent with observations of the oscillations in nonmagnetic regions, rather than magnetic ones. The main reason for this result could be our simplified treatment of the magnetic fields, which in our model are always uniform and either vertical or horizontal.

\section{Conclusions}

In this paper, we numerically simulated the propagation of MAWs in the gravitationally stratified solar atmosphere that is permeated by a uniform (horizontal or vertical) magnetic field. The computational model is structured by a realistic vertical temperature profile (Avrett \& Loeser 2008) but it is invariant along transversal and horizontal directions. The former allows us to simulate only MAWs but not Alfvén waves. Our studies also do not allow for internal gravity waves as they cannot propagate in the vertical direction. Magnetoacoustic waves are excited by a driver that is periodic in time, and acts upon a vertical component of velocity. The chosen wave periods of this driver are close to the values of the corresponding characteristics of the solar granulation, while the amplitude was set up to be very small to minimize contribution from nonlinear effects, which become pronounced in the simulations at higher altitudes anyway. 
Both horizontal and vertical magnetic fields are considered. In both cases, the field is assumed to be uniform. In the former case, only fast MAWs are generated, while the vertical field guides both slow and fast MAWs. The atmosphere filters the resulting waves whose periods vary with atmospheric height; waves with short wave periods are free to reach the solar corona, while waves with long wave periods become evanescent at those heights, at which $P$ is larger than the local cutoff wave period, evaluated analytically and resulting from the corresponding Lamb (Klein-Gordon) equations (e.g., Lamb 1910; Stark \& Musielak 1993; Roberts 2006).

Our main findings can be summarized as follows. We found the maximal period, $P_{\max }$, for the excited MAWs in the horizontal magnetic field to be $P_{\max }=230 \mathrm{~s}$, and in the vertical magnetic field $P_{\max }=255 \mathrm{~s}$. The waves with their wave periods larger than $P_{\max }$ are filtered by the solar atmosphere to the waves whose wave periods are shorter that the corresponding values of $P_{\max }$. Specifically, in the horizontal and vertical magnetic field cases, these shorter wave periods are $210 \mathrm{~s}$ and $227 \mathrm{~s}$, respectively. It is interesting that these wave periods are close to $3 \mathrm{~min}$, which is commonly observed in magnetically free regions of the solar chromosphere.

Our theoretical results significantly supplement and extend previous work on driving the solar atmospheric oscillations by acoustic and magnetic flux tube waves, and make theoretical predictions of variations of the cutoff frequencies for MHD waves with atmospheric heights. These theoretical predictions can be verified by future observations.

Acknowledgements. The authors express their thanks to unknown referees for their stimulating comments, to Dr. Piotr Konkol for his assistance in drawing certain figures, and to Dr. Pradeep Kayshap for discussions. This work was done in the framework of the project from the Polish Science Center (NCN) Grant No. 2014/15/B/ST9/00106. Z.E.M. acknowledges support of this work by the Alexander von Humboldt Foundation. The software used in this work was in part developed by the DOE-supported ASCI/Alliance Center for Astrophysical Thermonuclear Flashes at the University of Chicago. Numerical simulations were performed on the LUNAR/SOLARIS cluster at Institute of Mathematics of University of M. Curie-Skłodowska, Lublin, Poland.

\section{References}

Avrett, E. H., \& Loeser, R. 2008, ApJS, 175, 229

Bogdan, T. J., Carlsson, M., Hansteen, V. H., et al. 2003, ApJ, 599, 626

Campos, L. M. B. C. 1986, Rev. Mod. Phys., 58, 117

Chae, J., Cho, K., Song, D., et al. 2018, ApJ, 854, 127

Cirtain, J. W., Del Zanna, G., DeLuca, E. E., et al. 2007, ApJ, 655, 598

Collins, W. 1989a, ApJ, 337, 548

Collins, W. 1989b, ApJ, 343, 499

Dame, L. 1983, PhD Thesis, Université de Paris VII

De Pontieu, B., McIntosh, S. W., Carlsson, M., et al. 2007, Sci., 318, 1574

Deubner, F. L. 1991, in Mechanisms of Chromospheric and Coronal Heating, Proceedings of the International Conference, Heidelberg, 5-8 June 1990, XV eds. P. Ulmschneider, E. R. Priest, \& R. Rosner (Berlin, Heidelberg, New York: Springer-Verlag), 6

Erdélyi, R., \& Fedun, F. 2007, Sci., 318, 1572

Felipe, T., Khomenko, E., \& Collados, M. 2010, ApJ., 719, 357

Felipe, T., Khomenko, E., \& Collados, M. 2011, ApJ, 735, 65

Felipe, T., Socas-Navarro, H., \& Khomenko, E. 2014, ApJ, 795, 9

Fleck, B., \& Schmitz, F. 1991, A\&A, 250, 235

Fleck, B., \& Schmitz, F. 1993, A\&A, 273, 671

Fujimura, D., \& Tsuneta, S. 2009, ApJ, 702, 1443

Gruszecki, M., Murawski, K., \& McLaughlin, J. A. 2008, A\&A, 489, 413

Gruszecki, M., Murawski, K., Fromang, S., et al. 2009, Acta Phys. Polon. B, 40, 367

Hasan, S. S., van Ballegooijen, A. A., Kalkofen, W., et al. 2005, ApJ, 631, 1270

Jess, D. B., Mathioudakis, M., Erdélyi, R., et al. 2009, Sci, 323, 1582

Kalkofen, W. 1997, ApJ, 486, 145

Kalkofen, W., Rossi, P., Bodo, G., et al. 1994, A\&A, 284, 976

Khomenko, E., \& Collados, M. 2015, Liv. Rev. Sol. Phys., 12, 6

Khomenko, E., \& Cally, P. S. 2012, ApJ, 746, 68
Khomenko, E., \& Collados, M. 2012, ApJ, 747, 87

Khomenko, E., Collados, M., \& Felipe, T. 2008, Sol. Phys., 251, 589

Konkol, P., \& Murawski, K. 2010, Acta Phys. Polon., 41, 1369

Konkol, P., Murawski, K., Lee, D., et al. 2010, A\&A, 521, A34

Kuperus, M. 1966, IAUTB, 12, 564

Kuźma, B., Murawski, K., Zaqarashvili, T. V., et al. 2017a, A\&A, 597, A133

Kuźma, B., Murawski, K., Kayshap, P., et al. 2017b, ApJ, 849, 78

Lamb, H. 1909, Proc. Lond. Math. Soc., 7, 122

Lamb, H. 1910, Proc. R. Soc. London A, 34, 551

Lamb, H. 1945, Hydrodynamics (New York: Dover Publications)

Lee, D. 2013, J. Comp. Phys., 243, 269

Lee, D., \& Deane, A. E. 2009, J. Comp. Phys., 228, 952

Leibacher, J., \& Stein, R. F. 1971, ApJ, 7, L191

Leroy, B. 1981, A\&A, 97, 245

Lighthill, M. J. 1952, Proc. Royal Soc. London, A211, 564

McAteer, R. T., Gallagher, P. T., Williams, D. R., et al. 2002, ApJ, 567, L165

McAteer, R. T., Gallagher, P. T., Williams, D. R., et al. 2003, ApJ, 587, 806

Morgan, H., \& Taroyan, Y. 2017, Sci. Adv., 3, e1602056

Murawski, K. 1992, Sol. Phys., 139, 279

Murawski, K. 2002, Analytical and Numerical Methods for Wave Propagation in Fluids (Singapore: World Scientific)

Murawski, K., \& Musielak, Z. 2016, MNRAS, 463, 4433

Murawski, K., \& Zaqarashvili, T. V. 2010, A\&A, 519, A8

Murawski, K., Nocera, L., \& Mędrek, M. 2001, A\&A, 376, 708

Musielak, Z. E. 1986, J. Plasma Phys., 36, 341

Musielak, Z. E. 1990, ApJ, 351, 287

Musielak, Z. E., \& Rosner, R. 1987, ApJ, 315, 371

Musielak, Z. E., \& Rosner, R. 1988, ApJ, 329, 376

Musielak, Z. E., \& Ulmschneider, P. 2003a, A\&A, 400, 1057

Musielak, Z.E., \& Ulmschneider, P. 2003b, A\&A, 406, 725

Musielak, Z. E., Moore, R. L., Suess, S. T., et al. 1989, ApJ, 344, 478

Musielak, Z. E., Fontenla, J. M., \& Moore, R. L. 1992, Phys. Fluids B, 4, 13

Nakariakov, V. N., Roberts, B., \& Murawski, K. 1997, Sol. Phys., 175, 93

Nakariakov, V. N., Roberts, B., \& Murawski, K. 1998, A\&A, 332, 795

Nye, A. H., \& Thomas, J. H. 1974, Sol. Phys., 38, 399

Ogrodowczyk, R., Murawski, K., \& Solanki, S. K. 2009, A\&A, 495, 313

Okamoto, T. J., \& De Pontieu, B. 2011, ApJ, 736, L24

Osterbrock, D. E. 1961, ApJ, 134, 347

Parker, E. N. 1964, ApJ, 140, 1170

Parker, E. N. 1979, Cosmical Magnetic Fields: Their Origin and Their Activity

(New York: Oxford University Press)

Petukhov, M Yu, \& Petukhov, Yu V 2003, Astron. Lett., 29, 116

Priest, E. R. 2014, Magnetohydrodynamics of the Sun (Cambridge: Cambridge University Press)

Rae, I. C., \& Roberts, B. 1982, ApJ, 256, 761

Roberts, B. 2006, Phil. Trans. R. Soc. A, 364, 447

Rossi, B., \& Olbert, S. 1970, Introduction to the Physics of Space (New York: McGraw-Hill)

Santamaria, I. C., Khomenko, E., \& Collados, M. 2015, A\&A, 577, A70

Santamaria, I. C., Khomenko, E., Collados, M., et al. 2016, A\&A, 590, A3

Schmitz, F., \& Fleck, B. 1992, A\&A, 260, 447

Schmitz, F., \& Fleck, B. 1995, A\&A, 301, 483

Selwa, M., Murawski, K., Solanki, S. K., et al. 2005a, A\&A, 440, 385

Selwa, M., Murawski, K., \& Solanki, S. K. 2005b, A\&A, 436, 701

Selwa, M., Solanki, S. K., Murawski, K., et al. 2006, A\&A, 454, 653

Selwa, M., Murawski, K., Solanki, S. K., et al. 2007, A\&A, 462, 1127

Stark, B. A., \& Musielak, Z. E. 1993, AJ, 409, 450

Sutmann, G., \& Ulmschneider, P. 1995a, A\&A, 294, 232

Sutmann, G., \& Ulmschneider, P. 1995b, A\&A, 294, 241

Sutmann, G., Musielak, Z. E., \& Ulmschneider, P. 1998, A\&A, 340, 556

Tarr, L. A., Linton, M., \& Leake, J. 2017, ApJ, 837, 94

Thomas, J. H. 1982, ApJ, 262, 760

Thomas, J. H. 1983, Ann. Rev. Fluid Mech., 15, 321

Toro, E. 2009, Riemann Solvers and Numerical Methods for Fluid Dynamics (Berlin: Springer)

Tritschler, A., Schlichenmaier, R., \& Bellot Rubio, L. R. 2005, American Geophysical Union, Spring Meeting 2005, abstract No. SP11A-08

Ulmschneider, P., \& Stein, R. F. 1982, A\&A, 106, 9

Ulmschneider, P., Schmitz, F., Kalkofen, W., et al. 1978, A\&A, 70, 487

Ulmschneider, P., Musielak, Z. E., \& Fawzy, D. E. 2001, A\&A, 374, 662

Ulrich, R. K. 1970, ApJ, 162, 993

Van Doorsselaere, T., Nakariakov, V. M., \& Verwichte, E. 2008, A\&A, 485, 849

Wang, T. J., \& Solanki, S. K. 2004, A\&A, 421, 33

Wasiljew, A., \& Murawski, K. 2009, A\&A, 498, 863

Zhugzhda, Iu D, \& Dzhalilov, N. S. 1984a, A\&A, 132, 45

Zhugzhda, Iu D, \& Dzhalilov, N. S. 1984b, A\&A, 132, 52

Zhugzhda, Iu D, \& Dzhalilov, N. S. 1984c, A\&A, 133, 333 